\title{
Gastrointestinal stromal tumor (GIST)
}

\author{
Manoel de Souza Rocha*
}

In the course of the last decade there have been many developments in the recognition and treatment of gastrointestinal stromal tumors (GISTs). However, the most significant of these developments has been the discovery of a specific treatment for this neoplasm.

Initially utilized in the treatment for chronic myeloid leukemia, the imatinib mesylate has started being tested for GISTs so successfully that it has led to the immediate publication of a study in a case report format, by the New England Journal of Medicine ${ }^{(1)}$, something absolutely unusual in this widely regarded as the most reputable medical journal in the world.

GISTs are rare neoplasms, representing about $1 \%$ of gastrointestinal tumors, although this number may be underestimated.

But, what does make these tumors important for radiologists?

As demonstrated by the study published in the present issue of Radiologia Brasileira $^{(2)}$, the majority of GISTs are exophytic, which makes their diagnosis more difficult.

Therefore, cross-sectional imaging methods play a significant role in the identification of neoplastic masses and allow the recognition of their sites of origin.

The staging of these neoplasms is also based on diagnostic imaging methods, and radiologists should be familiar with some peculiarities of this disease, such as the fact that these tumors exceptionally, or never according to some authors, present lymph node metastasis.

Another very peculiar characteristic of GISTs is that from their emergence, or most usually, after the introduction of the imatinib therapy, metastases may present a "cystic" aspect ${ }^{(3)}$.

Although the aggressiveness of these tumors is variable and difficult to predict, it seems that there is a correlation between computed tomography finding of tumor necrosis and a worst prognosis, in comparison with more homogeneous tumors.

Sometimes, metastases with a positive response to therapy may present an increase in volume at tomography. So, the radiologist must be attentive to the internal pattern of the lesion, because in case of the disease relapsing, solid nodules may be seen scattered throughout the tumor liquefaction.

The most recent literature has reported that it seems there is a good correlation between computed tomography findings of GISTs after imatinib treatment demon-

* PhD, Docent at Department of Radiology, Faculty of Medicine - Universidade de São Paulo, São Paulo, SP, Brazil. E-mail: manoelrocha@usp.br 
strating a decrease in attenuation of liver metastases and the decrease in the metabolic activity detected on PET-CT ${ }^{(4)}$.

Older radiologists remember that, in the past, many of these gastrointestinal stromal tumors were diagnosed as leiomyomas or leiomyosarcomas. The new immuno-histochemical staining techniques allow recognizing that, in truth, the greatest majority of gastrointestinal mesenchymal tumors are GISTs, but a lower percentage of tumors correspond to true leiomyomas or leiomyosarcomas. Such differentiation is not feasible without the immuno-histochemical staining technique. Therefore, our role as investigators of macroscopic alterations is to identify an expansive lesion of deep wall layers of a gastrointestinal segment and to indicate the likelihood of a mesenchymal tumor. The final identification of the type of tumor requires an anatomopathological study including immuno-histochemical staining techniques.

\section{References}

1. Joensuu H, Roberts PJ, Sarlomo-Rikala M, et al. Effect of the tyrosine kinase inhibitor STI571 in a patient with a metastatic gastrointestinal stromal tumor. N Engl J Med 2001;344:1052-1056.

2. Macedo LL, Torres LR, Faucz RA, et al. Tumor do estroma gastrintestinal: achados clínicos, radiológicos e anatomopatológicos. Radiol Bras 2007;40:149-153.

3. Chen MYM, Bechtold RE, Savage PD. Cystic changes in hepatic metastases from gastrointestinal stromal tumor (GISTs) treated with Gleevec (imatinib mesylate). AJR Am J Roentgenol 2002;179: 1059-1062.

4. Choi H, Charnsangavej C, Faria SC, et al. Correlation of computed tomography and positron emission tomography in patients with metastatic gastrointestinal stromal tumor treated at a single institution with imatinib mesylate: proposal of new computed tomography response criteria. J Clin Oncol $2007 ; 25: 1753-1759$. 